\title{
Effects of different blood-pressure-lowering regimens on major cardiovascular events: results of prospectively-designed overviews of randomised trials
}

\author{
Blood Pressure Lowering Treatment Trialists' Collaboration*
}

\begin{abstract}
Summary
Background The benefits of reducing blood pressure on the risks of major cardiovascular disease are well established, but uncertainty remains about the comparative effects of different blood-pressure-lowering regimens. We aimed to estimate effects of strategies based on different drug classes (angiotensin-converting-enzyme [ACE] inhibitors, calcium antagonists, angiotensin-receptor blockers [ARBs], and diuretics or $\beta$ blockers) or those targeting different blood pressure goals, on the risks of major cardiovascular events and death.
\end{abstract}

Methods We did seven sets of prospectively-designed overviews with data from 29 randomised trials ( $n=162341$ ). The trial eligibility criteria, primary outcomes, and main hypotheses were specified before the result of any contributing trial was known.

\begin{abstract}
Findings In placebo-controlled trials the relative risks of total major cardiovascular events were reduced by regimens based on ACE inhibitors $(22 \%$; $95 \% \mathrm{Cl} 17-27)$ or calcium antagonists (18\%; 5-29). Greater risk reductions were produced by regimens that targeted lower blood pressure goals (15\%; 5-24). ARB-based regimens reduced the risks of total major cardiovascular events (10\%; 4-17) compared with control regimens. There were no significant differences in total major cardiovascular events between regimens based on ACE inhibitors, calcium antagonists, or diuretics or $\beta$ blockers, although ACE-inhibitor-based regimens reduced blood pressure less. There was evidence of some differences between active regimens in their effects on cause-specific outcomes. For every outcome other than heart failure, the difference between randomised groups in achieved blood pressure reduction was directly related to the observed difference in risk.
\end{abstract}

Interpretation Treatment with any commonly-used regimen reduces the risk of total major cardiovascular events, and larger reductions in blood pressure produce larger reductions in risk.

Lancet 2003; 362: 1527-35

\section{Introduction}

Cardiovascular diseases are the leading cause of death worldwide; ${ }^{1}$ about two-thirds of the cerebrovascular disease burden and half the ischaemic heart disease burden are attributable to non-optimum blood pressure. ${ }^{2}$ The beneficial effects of blood-pressure-lowering treatments on the risks of major cardiovascular events are well established, ${ }^{3-8}$ but little is known about the comparative effects of regimens based on different drug classes or regimens targeting different blood pressure goals. ${ }^{4,9,10}$ Reliable information about the size of benefits achieved with different blood-pressure-lowering regimens is of great importance-if one regimen proved even slightly better than another, then preferential use of the more effective regimen might prevent tens of thousands of major cardiovascular events every year.

The uncertainty about the comparative effects of different regimens in part reflects the limited statistical power of most individual studies to identify plausible differences in the size of treatment effects, and differences between studies in selection of patients, choice of outcome definitions, and achieved blood pressure reductions. The Blood Pressure Lowering Treatment Trialists' Collaboration was established to undertake a series of prospectively-designed systematic overviews to investigate the effects of different blood-pressure-lowering regimens on mortality and major cardiovascular events. ${ }^{11}$ Results of the first round of analyses were reported in $2000 .{ }^{4}$ The first meta-analyses showed benefits of bloodpressure-lowering regimens based on angiotensinconverting-enzyme (ACE) inhibitors and those based on calcium antagonists, compared with placebo, but did not provide definitive evidence about comparative effects of regimens based on different drug classes and those targeting different blood pressure goals. In these analyses we aimed to resolve some of these issues.

\section{Methods}

The methods used by the Blood Pressure Lowering Treatment Trialists' Collaboration have been reported previously, , $^{411}$ and only the main components are summarised here. Trial eligibility criteria, blood pressure lowering regimens to be compared, and primary outcomes were all prespecified. ${ }^{11}$

\section{Trial eligibility}

Inclusion criteria were: 1) random allocation of patients to either a blood-pressure-lowering drug or placebo; 2) random allocation of patients to different blood pressure goals; or 3) random allocation of patients to regimens based on different classes of blood-pressure-lowering drug. Trials were also required to have a planned minimum of 1000 patient-years' follow-up in each randomised group, and their main results should not have been reported before the overview protocol was finalised in July, 1995. In this and the previous round of analyses, ${ }^{4}$ overviews were
Correspondence to: Dr Fiona Turnbull, Blood Pressure Lowering Treatment Trialists' Collaboration, The George Institute for International Health, University of Sydney, PO Box 576, Newtown, Sydney, New South Wales 2042, Australia (e-mail: fturnbull@iih.usyd.edu.au) 
done if more than one trial were eligible for any comparison. Trials that included patients who were selected mainly on the basis of high blood pressure, diabetes mellitus, coronary heart disease, peripheral vascular disease, cerebrovascular disease, or renal disease, were eligible for inclusion. Investigations of patients who were selected mainly on the basis of other disorders, such as acute myocardial infarction or heart failure, were not included since most of these trials were part of other metaanalyses. For inclusion in our analyses, follow-up had to be complete and outcome data available by June, 2003.

\section{Comparisons and outcomes}

For these analyses, we did separate overviews of trials of: 1) ACE-inhibitor-based regimens versus placebo; 2) calcium-antagonist-based regimens versus placebo; 3) regimens targeting different blood pressure goals; 4) angiotensin-receptor blockers (ARB) regimens versus control regimens; 5) regimens based on ACE inhibitors versus regimens based on diuretics or $\beta$ blockers; 6) calcium-antagonist-based regimens versus regimens based on diuretics or $\beta$ blockers; and 7) ACE-inhibitor-based regimens versus calcium-antagonist-based regimens. ARB comparisons differed somewhat from other treatment comparisons and were therefore presented separately. One trial $^{12}$ was a placebo-controlled study in which active treatment was initiated in a large proportion of the placebo group early in the study (starting with diuretic-based regimens but with the addition of agents other than ACE inhibitors and ARBs, as required). Two trials ${ }^{13,14}$ used a placebo control while simultaneously attempting to achieve blood pressure reductions in both randomised groups (using blood-pressure-lowering agents other than ACE-inhibitors and the specific trial intervention

\begin{tabular}{|c|c|c|c|c|c|}
\hline & Treatment comparison & $\mathbf{n}$ & Trial design & Entry criteria* & $\begin{array}{l}\text { Follow-up } \\
\text { (years) }\end{array}$ \\
\hline \multicolumn{6}{|c|}{ Active treatment vs placebo } \\
\hline $\mathrm{HOPE}^{26}$ & Ramipril vs placebo & 9297 & DB & $\mathrm{CHD}, \mathrm{CVD}$, or $\mathrm{DM}+\mathrm{RF}$ & 4.5 \\
\hline PART2 $^{19}$ & Ramipril vs placebo & 617 & DB & CHD or CVD & $4 \cdot 7$ \\
\hline PROGRESS ${ }^{21}$ & Perindopril (+/- indapamide) vs placebo(s) & 6105 & DB & Cerebrovascular disease & 3.9 \\
\hline QUIET ${ }^{27}$ & Quinapril vs placebo & 1750 & DB & $\mathrm{CHD}$ & $2 \cdot 3$ \\
\hline $\mathrm{SCAT}^{22}$ & Enalapril vs placebo & 460 & $\mathrm{DB}$ & $\mathrm{CHD}$ & $4 \cdot 0$ \\
\hline \multicolumn{6}{|c|}{ Calcium antagonist vs placebo } \\
\hline IDNT $^{13}$ & Amlodipine vs placebo & 1136 & DB & HBP+DM+nephropathy & $2 \cdot 6$ \\
\hline $\mathrm{NICOLE}^{28}$ & Nisoldipine vs placebo & 826 & DB & $\mathrm{CHD}$ & 3.0 \\
\hline PREVENT $^{20}$ & Amlodipine vs placebo & 825 & DB & $\mathrm{CHD}$ & $3 \cdot 0$ \\
\hline SYST-EUR ${ }^{23}$ & Nitrendipine vs placebo & 4695 & DB & $\mathrm{HBP}, \geqslant 60$ years & $2 \cdot 6$ \\
\hline \multicolumn{6}{|c|}{ More intensive vs less intensive regimens } \\
\hline $\mathrm{AASK}^{29}$ & MAP $\leqslant 92$ vs $102-107 \mathrm{~mm} \mathrm{Hg}$ & 1094 & Open & HBP+nephropathy, Afr & $3 \cdot 8$ \\
\hline $\operatorname{ABCD}(\mathrm{H})^{30}$ & $\mathrm{DBP} \leqslant 75$ vs $\leqslant 90 \mathrm{~mm} \mathrm{Hg}$ & 470 & Open & $\mathrm{HBP}+\mathrm{DM}$ & $5 \cdot 3$ \\
\hline $\operatorname{ABCD}(\mathrm{N})^{31}$ & DBP 10 below baseline vs $80-89 \mathrm{~mm} \mathrm{Hg}$ & 480 & Open & $\mathrm{DM}$ & $5 \cdot 3$ \\
\hline HOT $\neq^{17}$ & $\mathrm{DBP} \leqslant 80$ vs $\leqslant 85$ or $\leqslant 90 \mathrm{~mm} \mathrm{Hg}$ & 18790 & Opent & HBP & $3 \cdot 8$ \\
\hline UKPDS-HDS ${ }^{24}$ & DBP $<85$ vs $<105 \mathrm{~mm} \mathrm{Hg}$ & 1148 & Open & HBP+DM & $8 \cdot 4$ \\
\hline \multicolumn{6}{|c|}{ ARBs vs control regimens } \\
\hline IDNT $^{13}$ & Irbesartan vs placebo§ & 1148 & DB & HBP+DM +nephropathy & $2 \cdot 6$ \\
\hline RENAAL $^{14}$ & Losartan vs placebo§ & 1513 & DB & $\mathrm{DM}+$ nephropathy & $3 \cdot 4$ \\
\hline SCOPE $^{12}$ & Candesartan vs placebo§ & 4937 & DB & HBP, $70-89$ years & 4.5 \\
\hline LIFE $^{15}$ & Losartan vs atenolol & 9193 & DB & $\mathrm{HBP}+\mathrm{CVD} \mathrm{RF}$ & $4 \cdot 8$ \\
\hline \multicolumn{6}{|c|}{ Different drug classes } \\
\hline \multicolumn{6}{|c|}{ ACE inhibitor vs diuretic or $\beta$ blocker ${ }^{29}$} \\
\hline AASK $^{29}$ & Ramipril vs metoprolol & 877 & DB & HBP+nephropathy, Afr & $4 \cdot 1$ \\
\hline ALLHAT $^{32}$ & Lisinopril vs chlorthalidone & 24328 & DB & $\mathrm{HBP}+\mathrm{RF}$ & 4.9 \\
\hline ANBP2 $2^{33}$ & Enalapril vs hydrochlorothiazide & 6083 & Opent & HBP, 65-84 years & $4 \cdot 1$ \\
\hline CAPPP $^{34}$ & Captopril vs $\beta$ blocker or diuretic & 10985 & Opent & HBP & $6 \cdot 1$ \\
\hline STOP- $2^{35}$ & $\begin{array}{l}\text { Enalapril or lisinopril vs atenolol or metoprolol or } \\
\text { pindolol or hydrochlorothiazide+amiloride }\end{array}$ & 4418 & Opent & HBP, $70-84$ years & $5 \cdot 0$ \\
\hline UKPDS-HDS ${ }^{44}$ & Captopril vs atenolol & 758 & DB & $\mathrm{HBP}+\mathrm{DM}$ & $8 \cdot 4$ \\
\hline \multicolumn{6}{|c|}{ Calcium antagonist vs diuretic or $\beta$ blocker } \\
\hline AASK $^{29}$ & Amlodipine vs metoprolol & 658 & DB & HBP+nephropathy, Afr & $3 \cdot 0$ \\
\hline ALLHAT $^{32}$ & Amlodipine vs chlorthalidone & 24321 & DB & $\mathrm{HBP}+\mathrm{RF}$ & 4.9 \\
\hline CONVINCE ${ }^{36}$ & COER-verapamil vs hydrochlorothiazide or atenolol & 16476 & DB & $\mathrm{HBP}+\mathrm{RF}$ & $3 \cdot 0$ \\
\hline ELSA $^{37}$ & Lacidipine vs atenolol & 2334 & DB & HBP & $4 \cdot 0$ \\
\hline INSIGHT ${ }^{38}$ & Nifedipine GITS vs hydrochlorothiazide+amiloride & 6321 & DB & $\mathrm{HBP}+\mathrm{RF}$ & $4 \cdot 0$ \\
\hline NICS-EH ${ }^{18}$ & Nicardipine $v s$ trichlormethiazide & 429 & $\mathrm{DB}$ & $\mathrm{HBP}, \geqslant 60$ years & $5 \cdot 0$ \\
\hline NORDIL $^{39}$ & Diltiazem vs $\beta$ blocker or diuretic & 10881 & Opent & HBP & $5 \cdot 0$ \\
\hline $\mathrm{SHELL}^{40}$ & Lacidipine vs chlorthalidone & 1882 & Open & $\mathrm{HBP}, \geqslant 60$ years & 3.6 \\
\hline STOP- $2^{35}$ & $\begin{array}{l}\text { Felodipine or isradipine vs atenolol or metoprolol } \\
\text { or pindolol or hydrochlorothiazide+amiloride }\end{array}$ & 4409 & Opent & HBP, $70-84$ years & $5 \cdot 0$ \\
\hline VHAS $^{25}$ & Verapamil vs chlorthalidone & 1414 & Open & HBP & $2 \cdot 0$ \\
\hline \multicolumn{6}{|c|}{$\overline{\text { ACE inhibitor vs calcium antagonist }}$} \\
\hline AASK $^{29}$ & Ramipril vs amlodipine & 653 & DB & $\mathrm{HBP}+$ nephropathy, Afr & $3 \cdot 0$ \\
\hline $\operatorname{ABCD}(\mathrm{H})^{30}$ & Enalapril vs nisoldipine & 470 & DB & $\mathrm{HBP}+\mathrm{DM}$ & $5 \cdot 3$ \\
\hline $\operatorname{ABCD}(\mathrm{N})^{31}$ & Enalapril vs nisoldipine & 480 & DB & $\mathrm{DM}$ & $5 \cdot 3$ \\
\hline ALLHAT $^{32}$ & Lisinopril vs amlodopine & 18113 & DB & $\mathrm{HBP}+\mathrm{CVD} \mathrm{RF}$ & 4.9 \\
\hline $\mathrm{JMIC}^{41}$ & ACE inhibitor vs nifedipine & 1650 & Opent & $\mathrm{HBP}+\mathrm{CHD}$ & $3 \cdot 0$ \\
\hline STOP- $2^{35}$ & Enalapril or lisinopril vs felodipine or isradipine & 4401 & Opent & HBP, $70-84$ years & $5 \cdot 0$ \\
\hline
\end{tabular}

Afr=African American. $\mathrm{CHD}=$ coronary heart disease. $\mathrm{COER}=$ controlled onset, extended release. $\mathrm{CVD}=$ cardiovascular disease. $\mathrm{DB}=$ double-blind. $\mathrm{DBP}=$ diastolic blood pressure. $\mathrm{DM}=$ diabetes mellitus. GITS=gastrointestinal transport system. $\mathrm{HBP}=$ high blood pressure. MAP=mean arterial pressure. $\mathrm{RF}=\mathrm{other} \mathrm{CVD}$ risk factor. *Definitions of HBP and nephropathy varied between studies. †PROBE (Prospective, Randomised, Open with Blinded Endpoint evaluation) design trials. $\ddagger$ HOT trial data analysed as most intensively treated group vs others. §These placebo-controlled trials either had similar blood pressure goals in each randomised group or introduced active treatment into the placebo group for another reason for a large proportion of participants before the completion of follow-up.

Table 1: Characteristics of included trials 
treatments). One trial ${ }^{15}$ was designed as a head-to-head comparison between active agents. Since all these included control treatment with agents other than ARBs, we analysed them as one group. No overviews of regimens based on $\alpha$-adrenergic receptor blockers were undertaken because only one trial was eligible. ${ }^{16}$ For every comparison, we tested the null hypothesis of no difference between groups for all six predefined outcomes: ${ }^{4,11}$ stroke, coronary heart disease, heart failure, major cardiovascular events (ie, stroke, coronary heart disease, heart failure, or cardiovascular death), death from any cardiovascular cause, and total mortality. For our analyses, the definition of heart failure included only those events that caused death or led to admission to hospital.

\section{Data collection and statistical analyses}

For every trial, information about baseline characteristics, blood pressure during follow-up, and outcome events was obtained from individual participant datasets ${ }^{17-25}$ or from tabulated data ${ }^{12-15,26-41}$ provided by collaborating investigators. Data were checked for completeness and balance between randomised groups, and were reviewed for accuracy on at least two occasions. In several instances, ${ }^{17-19,22,23,38}$ data included in the first round of overviews $^{4}$ have been updated on the basis of new information. Mean levels of baseline characteristics and mean differences in follow-up blood pressure between randomised comparisons were calculated for groups of trials with estimates from every individual study weighted by the number of individuals in the study. Effects of randomised treatments were analysed with the metan routine (STATA Release, version 6.0). Relative risks and $95 \%$ CIs for every outcome were calculated separately for every trial, with grouped data, in intention-to-treat analyses. Overall estimates of effect were calculated with a fixed-effects model, in which the log relative risk for every trial was weighted by the reciprocal of the variance of the log relative risk..$^{42}$ The assumption of homogeneity between the treatment effects in different trials was tested with the $\chi^{2} \mathrm{Q}$ statistic. If the assumption of homogeneity was rejected $(p<0 \cdot 10),{ }^{43}$ additional analyses were done with a random effects model. ${ }^{43}$ Participants could contribute only one event to the calculation for each outcome, but could contribute one event to each of the separate analyses of different outcomes. Associations between the size of blood pressure reductions and the observed differences in risk between randomised comparisons were estimated by plotting pooled relative risk estimates (and 95\% CIs) for every comparison and outcome against the corresponding mean blood pressure differences weighted by the number of individuals in each treatment group. Since the estimates are not independent, no formal test of association was done.

\section{Role of the funding source}

These overviews were designed and reported by a consensus of members of the Blood Pressure Lowering Treatment Trialists' Collaboration. The National Health and Medical Research Council of Australia funded the central coordination, analysis, and report writing at The George Institute for International Health in Sydney, Australia. Meetings of collaborators in this meta-analysis were funded by several pharmaceutical companies. Sponsor representatives were able to attend these meetings but did not contribute to study design, analysis or interpretation of results, writing the report, nor did they influence the decision to submit the report for publication.

\section{Results}

\section{Trials and patients}

Table 1 shows characteristics of the 29 trials $^{12-15,17-41,44}$ (162341 participants) that were included. Nine trials (25711 participants, 3548 major vascular events) provided data from comparisons of an ACE-inhibitorbased or a calcium-antagonist-based regimen versus placebo, and five (21982 participants, 1191 major vascular events) provided data from comparisons of regimens targeting different blood pressure goals. Four trials (16791 participants, 2478 major vascular events) provided data from comparisons of blood-pressurelowering regimens based on ARBs versus control regimens. 16 trials that included 101228 participants and recorded 10131 major vascular events provided data from comparisons between active regimens based on ACE inhibitors, calcium-antagonists, or diuretics or $\beta$ blockers.

The mean duration of follow-up ranged from $2 \cdot 0$ to 8.4 years, representing more than 700000 patient-years. The overall mean age of trial participants was 65 years, $52 \%$ were men (table 2), and most were selected on the basis of having pre-existing cardiovascular disease or more than one cardiovascular risk factor at baseline. Participants' mean baseline blood pressure was $159 / 92 \mathrm{~mm} \mathrm{Hg}$, with mean values in the contributing trials ranging between 123 and $194 \mathrm{~mm} \mathrm{Hg}$ systolic and 74 and $106 \mathrm{~mm} \mathrm{Hg}$ diastolic. Table 2 shows composite mean blood pressures for different randomised comparisons

\begin{tabular}{|c|c|c|c|c|c|}
\hline \multirow[t]{2}{*}{ Treatment comparison } & \multirow[t]{2}{*}{$\begin{array}{l}\text { Age } \\
\text { (mean, years) }\end{array}$} & \multirow[t]{2}{*}{ Men } & \multirow[t]{2}{*}{$\begin{array}{l}\text { SBP/DBP at } \\
\text { entry (mean, } \mathrm{mm} \mathrm{Hg} \text { ) }\end{array}$} & \multicolumn{2}{|c|}{$\begin{array}{l}\text { Participants remaining on randomised treatment } \\
\text { or achieving blood pressure goal } \neq\end{array}$} \\
\hline & & & & 1st-listed & 2nd listed \\
\hline \multicolumn{6}{|l|}{ Active treatment vs placebo§ } \\
\hline ACEI vs placebo ${ }^{19,21,22,26,27}$ & 64 & $74 \%$ & $140 / 81$ & $73 \%$ & $75 \%$ \\
\hline CA vs placebo ${ }^{13,20,23,28}$ & 66 & $48 \%$ & $148 / 75$ & $74 \%$ & $69 \%$ \\
\hline \multicolumn{6}{|l|}{$\begin{array}{l}\text { More intensive vs less intensive } \\
\text { regimens }{ }^{24,29-31}\end{array}$} \\
\hline \multicolumn{6}{|l|}{ ARBs vs controls ${ }^{12-14,41}$} \\
\hline & 70 & $46 \%$ & $169 / 93$ & $65 \%$ & $58 \%$ \\
\hline \multicolumn{6}{|l|}{ Different drug classes } \\
\hline ACEI vs diuretic or $\beta$ blocker $24,29,32-35$ & 65 & $51 \%$ & $157 / 90$ & $63 \%$ & $72 \%$ \\
\hline CA vs diuretic or $\beta$ blocker ${ }^{18,29,29,32,35-40}$ & 66 & $48 \%$ & $159 / 92$ & $75 \%$ & $75 \%$ \\
\hline ACEI vs $\mathrm{CA}^{29-32,35,41}$ & 68 & $51 \%$ & $154 / 87$ & $63 \%$ & $71 \%$ \\
\hline
\end{tabular}

$\mathrm{ACE}=\mathrm{ACE}$ inhibitor. $\mathrm{CA}=$ calcium antagonist. $\mathrm{DBP}=$ diastolic blood pressure. $\mathrm{SBP}=$ systolic blood pressure. * For mean age, proportion of men, and mean $\mathrm{SBP} / \mathrm{DBP}$ at entry, overall mean values were calculated by weighting the values for every contributing trial by the number of individuals in that trial. For the proportion remaining on randomised treatment or achieving blood pressure goal, overall mean values for every treatment group were calculated by weighting the values for each group in every contributing trial by the number of individuals in that group. fProportion remaining on randomised treatment reported for all placebo-controlled studies and for studies comparing different active treatments. ‡Proportion achieving blood pressure goal reported for studies comparing more intensive with less intensive blood-pressure lowering regimens. §Data for adherence were not available for two trials; SCAT and IDNT.

Table 2: Characteristics of trial participants and adherence to randomised treatment during follow-up* 
trial. High blood pressure was the main inclusion criterion in 11 trials, but not in the remaining 18 (table 1).

Full information about the adherence of study participants to randomised treatments (table 2) was available from all but two trials. ${ }^{13,27}$ Overall, for most comparisons between a half and three-quarters of study participants remained on their randomly assigned study treatment until the end of follow-up. The proportion of participants achieving their target blood pressure in the groups assigned to more intensive blood-pressure lowering was a little over a half.

All but two small trials ${ }^{13,29}$ provided information about mean follow-up blood pressure differences between randomised groups. In the placebo-controlled trials of ACE-inhibitor-based or calcium-antagonist-based regimens, the weighted average blood pressure levels in treatment groups during follow-up were $5 \cdot 4 / 2 \cdot 3 \mathrm{~mm} \mathrm{Hg}$, and $8 \cdot 4 / 4 \cdot 2 \mathrm{~mm} \mathrm{Hg}$ lower, respectively, than in placebo groups (figure 1). In trials comparing regimens targeting different blood pressure goals, blood pressure was a mean of $4 \cdot 2 / 3.5 \mathrm{~mm} \mathrm{Hg}$ lower in groups assigned lower rather than higher targets. Follow-up blood pressure in groups assigned an ARB-based regimen were $2 \cdot 1 / 0 \cdot 9 \mathrm{~mm} \mathrm{Hg}$ lower than in those assigned control regimens (figure 2 ). In trials comparing treatment regimens, follow-up blood pressure in groups assigned ACE-inhibitor-based regimens were $1.9 / 0.2 \mathrm{~mm} \mathrm{Hg}$ higher than in those assigned diuretic-based or $\beta$-blocker-based regimens, and $0.6 / 0.9 \mathrm{~mm} \mathrm{Hg}$ higher than in those assigned calcium antagonists (figure 3). Follow-up blood pressure in groups assigned calcium-antagonist-based regimens were $0 \cdot 8 /$ $-0.2 \mathrm{~mm} \mathrm{Hg}$ higher than in those assigned to regimens based on diuretics or $\beta$ blockers. These differences in blood pressure should be taken into account in interpretation of our results for mortality and morbidity.

Most trials provided complete information about all six prespecified primary outcomes according to predefined criteria. However, one trial ${ }^{29}$ did not have information for any cause-specific outcomes, three ${ }^{12,27,28}$ had no heart failure data, and five had no information about heart failure as defined. ${ }^{23,34,35,38,39}$ One trial ${ }^{28}$ had no information about total major cardiovascular events or cardiovascular death. Data for coronary heart disease from five trials $^{13,14,22,28,36}$ did not include sudden death.

Further detailed information about the trials and analyses is available at http://www.iih.org/bplttc.

\section{Stroke}

ACE-inhibitor-based and calcium-antagonist-based regimens reduced the risk of stroke compared with placebo
Trials Events/participants Difference in BP* 1st listed 2nd listed (Mean, $\mathrm{mm} \mathrm{Hg}$ )

\section{Stroke}

ACEI vs placebo $19,21,22,26,275 \quad 473 / 9111 \quad 660 / 9118 \quad-5 /-2$

CA vs placebo $13,20,23,28$

More vs less ${ }^{17,24,30,31}$

Coronary heart disease

ACEI vs placebo ${ }^{19,21,22,26,27} 5 \quad 667 / 9111 \quad 834 / 9118 \quad-5 /-2$

CA vs placebo $13,20,23,28$

More vs less $17,24,30,31$

4 125/3794 156/3688 $-8 /-4$

Heart failure

ACEI vs placebo ${ }^{19,21,22,26,27} 5$

CA vs placebo $13,20,23,28$

4 274/7494 348/13394-4/-3

More vs less $30,31,35,43$

3 104/3382

$269 / 8246$

$-5 /-2$

Major cardiovascular events

ACEI vs placebo ${ }^{19,21,22,26,27} 5$ 1283/9111 1648/9118 -5/-2

CA vs placebo ${ }^{13,20,23,28}$

$3 \quad 280 / 3382 \quad 337 / 3274 \quad-8 /-4$

More vs less ${ }^{17,24,30,31}$

4 482/8034 719/13948-4/-3

Cardiovascular death

ACEI vs placebo ${ }^{19,21,22,26,27} 5 \quad 488 / 9111 \quad 614 / 9118 \quad-5 /-2$

CA vs placebo ${ }^{13,20,23,28}$

$4 \quad 107 / 3382135 / 3274 \quad-8 /-4$

More vs less ${ }^{17,24,29-31}$

$5209 / 8034271 / 13948-4 /-3$

\section{Total mortality}

ACEI vs placebo ${ }^{19,21,22,26,27} 5 \quad 839 / 9111 \quad 951 / 9118 \quad-5 /-2$

CA vs placebo $13,20,23,28$

$4239 / 3794263 / 3688 \quad-8 /-4$

More vs less ${ }^{17,24,29-31}$

5 404/8034 549/13948-4/-3

\section{Relative risk p (95\% Cl)}

$0.72(0.64-0.81) 0.33$

$0.62(0.47-0.82) 0.90$

$0.77(0.63-0.95) 0.15$

$0.80(0.73-0.88) 0.91$

$0.78(0.62-0.99) 0.34$

$0.95(0.81-1.11) 0.26$

$0.82(0.69-0.98) 0.60$

$1.21(0.93-1.58) 0.17$

$0.84(0.59-1.18) 0.11$

$0.78(0.73-0.83) 0.42$

$0.82(0.71-0.95) 0.54$

$0.85(0.76-0.95) 0.27$

$0.80(0.71-0.89) 0.29$

$0.78(0.61-1.00) 0.43$

$0.93(0.77-1.11) 0.15$

$0.88(0.81-0.96) 0.54$

$0.89(0.75-1.05) 0.99$

$0.96(0.84-1.09) 0.09$

$2 \cdot 0$

$\begin{array}{ccc}0.5 & 1 \cdot 0 & 2 \cdot 0 \\ \text { Relative risk } & \end{array}$

Favours 1st listed Favours 2nd listed

Figure 1: ACE inhibitors and calcium antagonists compared with placebo, and more and less intensive blood-pressure-lowering regimens $\mathrm{ACEl}=\mathrm{ACE}$ inhibitor. $\mathrm{CA}=$ calcium antagonist. More=more intensive blood-pressure-lowering regimen. Less=less intensive regimen. $\mathrm{p}$ values are derived from $\chi^{2}$ test for homogeneity. *Overall mean blood pressure difference (systolic/diastolic) during follow-up in the actively treated group compared with the control group, calculated by weighting the difference observed in each contributing trial by the number of individuals in the trial. Negative values indicate lower mean follow-up blood pressure in 1st listed groups (ACE, CA, and more) than in 2nd listed groups (placebo and less). 
(28\% [95\% CI 19-36]; and 38\% [18-53], respectively) (figure 1). Greater risk reductions were seen with regimens targeting lower blood pressure goals (23\% [5-37]) and the risk of stroke was reduced with ARB-based regimens compared with control regimens (21\% [10-31]; figure 2). In comparisons of regimens based on different drug classes, there were some differences in stroke risk, but these were of borderline significance (figure 3). There was a trend towards a greater risk reduction with regimens based on diuretics or $\beta$ blockers compared with regimens based on ACE inhibitors (9\% [0-18]), and trends towards greater reductions with regimens based on calcium antagonists compared with those based on diuretics or $\beta$ blockers (7\% [ -1 to 14$])$ or with those based on ACE inhibitors $(12 \%[1-25])$.

\section{Coronary heart disease}

ACE-inhibitor-based regimens reduced the risk of coronary heart disease (20\% [12-27]) compared with placebo (figure 1). There was weaker evidence of a reduction in risk with calcium-antagonist-based regimens $(22 \%$ [1-38]). For regimens targeting lower blood pressure goals, there was no clear evidence of benefit ( $5 \%$ [ -11 to 19$])$. There was also no clear difference between ARB-based regimens and control regimens ( $4 \%$ [ -9 to 15$]$ ) (figure 2). There was no significant difference between regimens based on ACE inhibitors, calcium antagonists, or diuretics or $\beta$ blockers, with narrow CIs for the estimate of effect for each comparison (figure 3). There was significant heterogeneity among trials that were included in the comparison of ACEinhibitor-based and calcium-antagonist-based regimens $(p=0 \cdot 01)$. Recalculation of the estimate and its $95 \%$ CIs with a random effects model made little difference $(17 \%$ [-5 to 32$])$.

\section{Heart failure}

For heart failure that caused death or admission to hospital, there was a beneficial effect from ACE inhibitorbased regimens compared with placebo (18\% [2-31]; figure 1). There was no clear effect of calcium-antagonistbased regimens $(21 \%$ [ -42 to 7$])$ or regimens targeting lower blood pressure goals ( $16 \%$ [ -18 to 41$]$ ), although the CIs were wide for both. Compared with control regimens, ARB-based treatment reduced the risk of heart failure (16\% [3-28]; figure 2). Effects of regimens based on ACE inhibitors did not differ significantly from effects of those based on diuretics or $\beta$ blockers ( $7 \%$ [ -4 to 19$]$; figure 3). However, compared with regimens based on calcium antagonists, those based on diuretics or $\beta$ blockers $(33 \%[21-47])$ and on ACE inhibitors (18\% [8-27]) produced greater reductions in risk.

\section{Major cardiovascular events}

Comparisons of regimens based on ACE inhibitors and calcium-antagonists with placebo both indicated significant reductions in the composite of all major cardiovascular events with active treatment $(22 \%$ [17-27]; and 18\% [5-29], respectively; figure 1). The risk of this outcome was also reduced with regimens targeting lower blood pressure goals (15\% [5-24]). ARB-based regimens reduced major cardiovascular events more than did control regimens $(10 \%$, [4- 17]; figure 2$)$. There were no significant differences between regimens based on ACE inhibitors, calcium antagonists, or diuretics or $\beta$ blockers, and CIs were narrow for the estimate for every comparison (figure 3 ).

\section{Cardiovascular death}

Compared with placebo, ACE-inhibitor-based regimens reduced the risk of cardiovascular death (20\% [11-29]) (figure 1). There was a trend towards fewer deaths with calcium-antagonist-based regimens (22\% [0-39]), but no clear evidence of a reduction in risk with regimens targeting low blood pressure goals (7\% [ -11 to 23$]$ ) or between ARB-based regimens and control regimens $(4 \%$ [ -8 to 15]; figure 2). Effects on cardiovascular mortality did not differ significantly between regimens based on ACE inhibitors, calcium-antagonists, or diuretics or $\beta$ blockers (figure 3).

\section{Total mortality}

In trials comparing ACE-inhibitor-based regimens and placebo, active treatment reduced the risk of death $(12 \%$ [4-19]; figure 1). There was no clear evidence of a difference in this outcome in trials comparing calciumantagonist-based regimens and placebo or in those comparing regimens targeting different blood pressure goals $(11 \%$ [ -5 to 25$]$ and $4 \%$ [-9 to 16$]$, respectively). Similarly, total mortality did not differ between ARBbased regimens and control regimens ( $6 \%$ [ -2 to 14$]$; figure 2) or between treatment regimens based on ACE inhibitors, calcium-antagonists, or diuretics or $\beta$ blockers

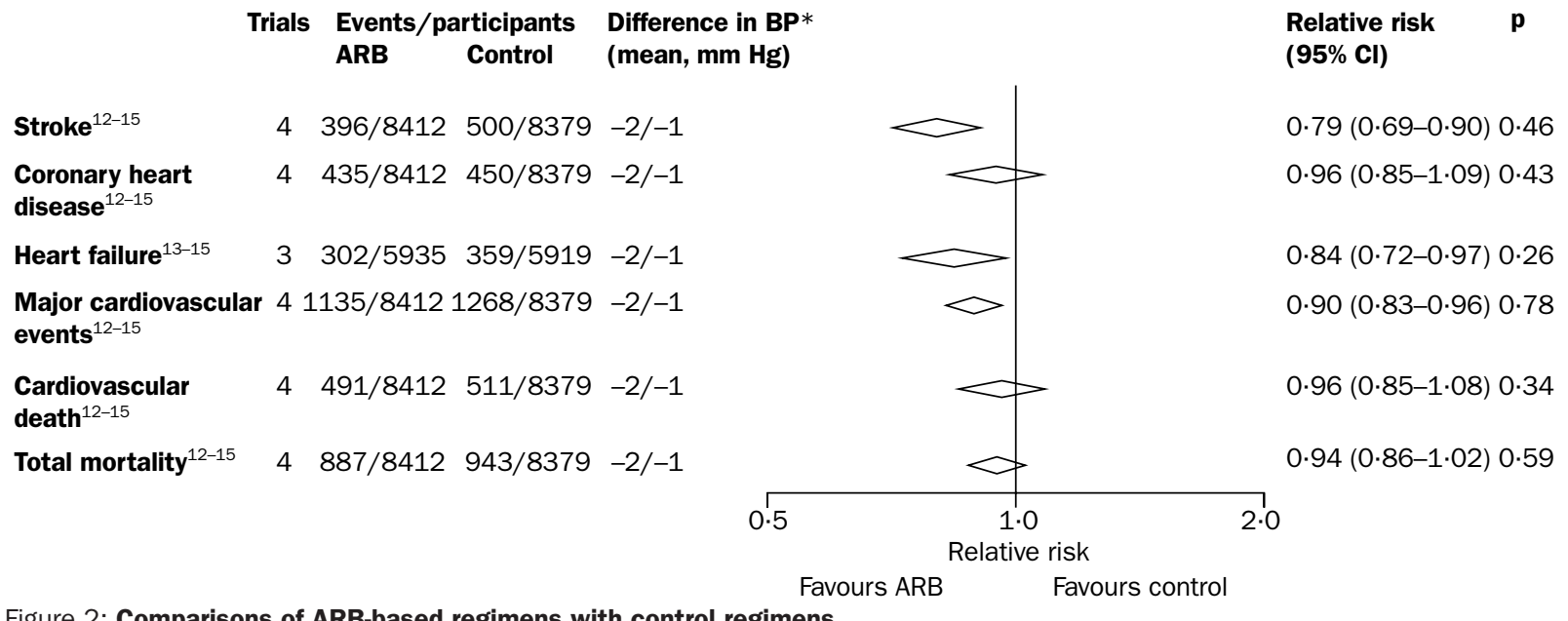

Figure 2: Comparisons of ARB-based regimens with control regimens

* Overall mean blood pressure difference (systolic/diastolic) during follow-up in the ARB group compared with the control group, calculated by weighting the difference observed in each contributing trial by the number of individuals in the trial. Negative values indicate lower mean follow-up blood pressure levels in the ARB group than in controls. $p$ values from $\chi^{2}$ test for homogeneity. 


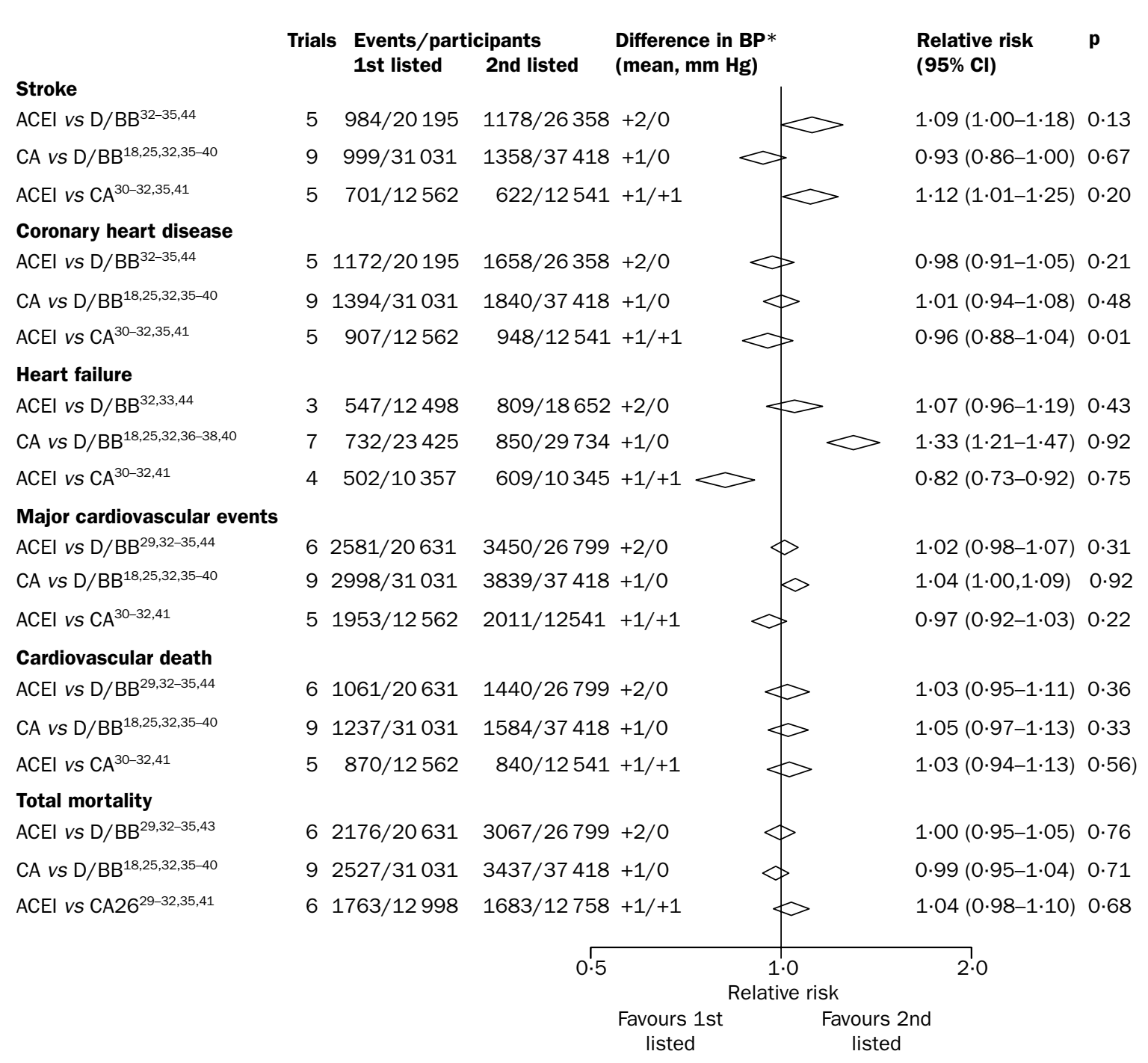

Figure 3: Comparisons of blood-pressure-lowering regimens based on different drug classes

$\mathrm{ACE}=\mathrm{ACE}$ inhibitor. $\mathrm{CA}=$ calcium antagonist. $\mathrm{D} / \mathrm{BB}=$ diuretic or $\beta$ blocker. $\mathrm{p}$ values from $\chi^{2}$ test for homogeneity. * Overall mean blood pressure difference (systolic/diastolic) during follow-up in the group assigned the first-listed treatment compared with the group assigned the second-listed treatment, calculated by weighting the difference observed in each contributing trial by the number of individuals in the trial. Positive values indicate a higher mean follow-up blood pressure in the 1st listed group (ACEI and CA) than in the 2nd listed group (D/BB and CA).

(figure 3). There was significant heterogeneity in trials that were included in the comparison of regimens targeting different blood pressure goals $(\mathrm{p}=0 \cdot 09)$. Recalculation of the estimate and its $95 \%$ CIs with a random effects model made little difference (11\% [-10 to 29$])$.

\section{Blood pressure differences and risk reductions}

For all seven randomised comparisons, the weighted mean blood pressure differences between randomised groups seemed to be directly associated with differences in risks of stroke, coronary heart disease, major cardiovascular events, cardiovascular death, and total mortality (figure 4). By contrast, blood pressure differences did not seem to be associated with risk of heart failure. In our present analyses, we did not investigate the separate association between blood pressure differences and outcome in trials of individual drug classes.

\section{Discussion}

Our results show that treatment with any commonly-used regimen reduces the risk of total major cardiovascular events, and that larger reductions in blood pressure produce larger reductions in risk. These results confirm earlier analyses ${ }^{4}$ showing benefits of ACE-inhibitor-based regimens across a wide range of hypertensive and nonhypertensive patients at high risk of cardiovascular disease, and of calcium-antagonist-based regimens in hypertensive patients.

The results of this meta-analysis also indicate differences between regimens based on different drugs in their effects on cause-specific cardiovascular outcomes. Although some of these differences seem to be largely explained by differences in achieved blood pressure, others seem to be independent of such differences. The results of previous overviews $^{4,9}$ and individual trials ${ }^{30,32-34}$ had raised this possibility, but until the completion of the present analyses, whether the findings were real was unknown.

Our results show that regimens based on ACE inhibitors or on diuretics or $\beta$ blockers are much more effective at preventing heart failure than are regimens based on calcium antagonists. Since the current analyses were restricted to cases of heart failure that resulted in death or admission to hospital, minor side-effects of calcium antagonists, such as peripheral oedema, are unlikely to be wholly responsible for this finding. The differences between these regimens are not easily 

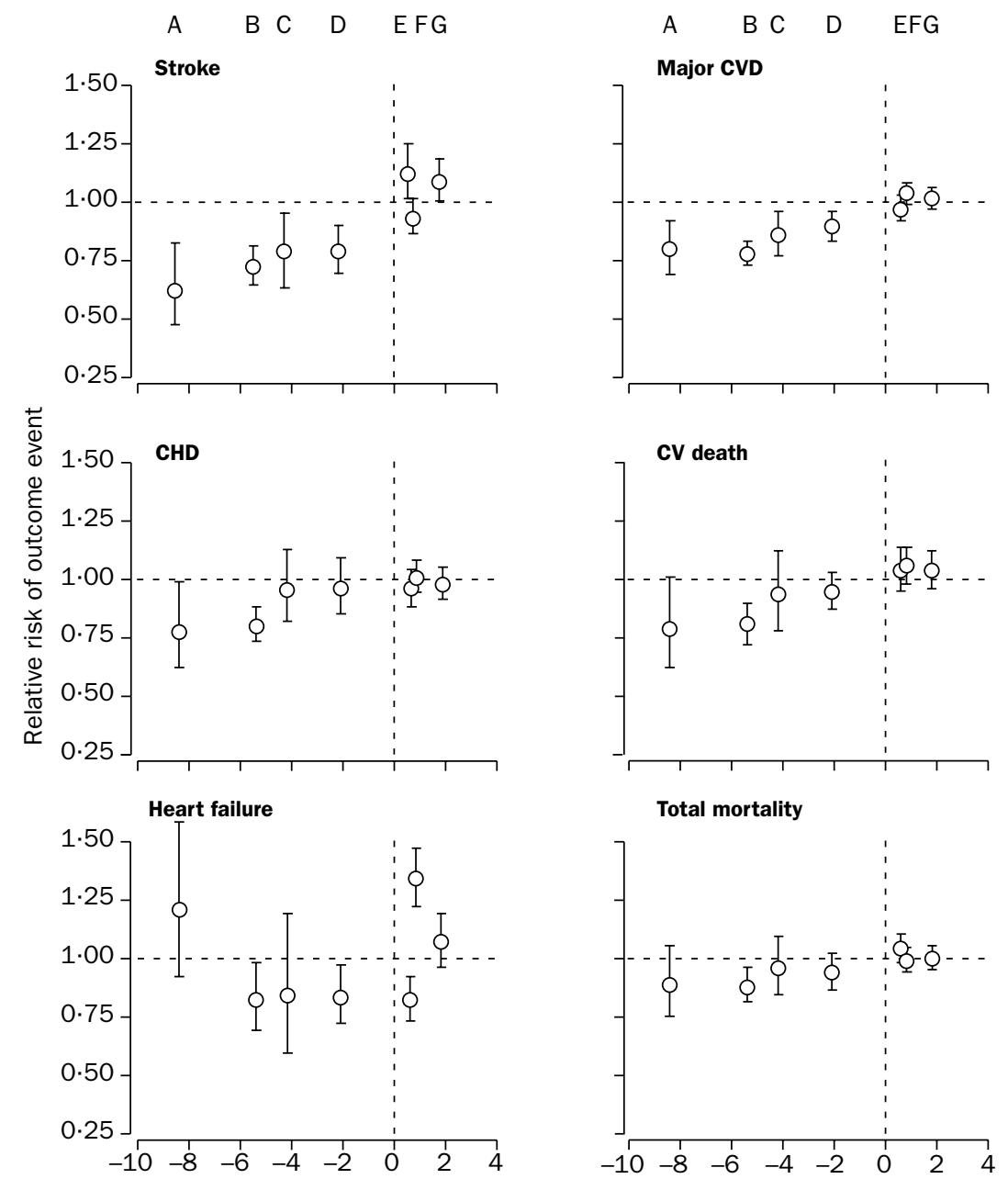

Systolic blood pressure difference between randomised groups ( $\mathrm{mm} \mathrm{Hg}$ )

Figure 4: Associations of blood pressure differences between groups with risks of major vascular outcomes and death

The circles are plotted at the point estimate of effect for the relative risk for every event type and the mean follow-up blood pressure in the 1 st listed group compared with the 2 nd listed group. Vertical lines are $95 \% \mathrm{Cls}$. $\mathrm{CHD}=$ coronary heart disease. $\mathrm{CVD}=$ cardiovascular event. $\mathrm{CV}$ death= cardiovascular death. A) calcium antagonist vs placebo, from references $13,20,23,28$. B) ACE inhibitor vs placebo, from references $19,21,22,26,27$. C) more intensive vs less intensive bloodpressure-lowering, from references 17, 24, 29-31. D) ARB vs control, from references 12-15. E) ACE inhibitor vs calcium antagonist, from references 29-32,35,41. F) calcium antagonist vs diuretic or $\beta$ blocker, from references $18,25,29,32,35-40$. G) ACE inhibitor vs diuretic and $\beta$ blocker from references $29,32-35$.

accounted for by their comparative effects on blood pressure and are broadly consistent with results of trials of ACE inhibitors ${ }^{7}$ and calcium antagonists ${ }^{45,46}$ in patients with heart failure. Our results also showed a greater effect of ARB-based regimens than other control regimens on the risk of heart failure, although this might, at least in part, be related to the greater reduction in blood pressure.

Unlike in earlier analyses, ${ }^{4}$ our results for coronary heart disease showed no trend towards a lesser effect of calcium antagonists compared with diuretics or $\beta$ blockers, or ACE inhibitors. Narrow CIs excluded all but a trivial difference in risk, thereby confirming conclusions from earlier reports, ${ }^{47,48}$ questioning the validity of claims of large increases in coronary risk in hypertensive patients treated with calcium antagonists. Our results showed no clear difference in risk of coronary heart disease between regimens based on ACE inhibitors and those based on diuretics or $\beta$ blockers, despite somewhat smaller blood pressure reductions with ACE inhibitors than with diuretics or $\beta$ blockers. Conversely, we noted no clear difference in risk of coronary heart disease between ARB-based and control regimens, despite greater blood pressure reductions with ARBs.

For stroke, we observed a greater effect of regimens based on calcium antagonists compared with those based on diuretics or $\beta$ blockers, but the result was of borderline significance. We also noted a trend towards greater reductions in stroke risk with regimens based on calcium antagonists and diuretics or $\beta$-blockers than with regimens based on ACE inhibitors. A greater reduction in stroke risk was noted with ARB-based regimens compared with control regimens that were less effective in reducing blood pressure. For all these differences there remains some uncertainty as to whether they can be accounted for entirely by differences in achieved blood pressure levels.

Although modest independent effects of specific drug classes on stroke or risk of coronary heart disease are not precluded by our findings, the results do suggest that blood pressure lowering is a major component of the benefit conferred by the regimens investigated. Direct evidence is provided by the regimens targeting different blood pressure goals (with various drug classes), which showed larger reductions in stroke and total major cardiovascular events from regimens targeting lower blood pressure goals. Indirect evidence is provided by the association of differences in blood pressure between randomised groups with differences in risks of stroke, coronary heart disease, and the composite outcomes of total major cardiovascular events and death (figure 4). For heart failure, however, blood pressure differences and the relative risk of this outcome were not clearly associated. This seemed to result, at least in part, from the divergent effects of calcium-antagonist-based regimens on this outcome-an effect that seemed to be determined mainly by factors other than reduction of blood pressure. A similar observation has been made with regard to the effect of the $\alpha$-adrenergic blocker, doxazosin, on the risk of heart failure. ${ }^{16}$

Combination of data from multiple trials addressing the same question reduces random errors and increases the precision of estimates of treatment effects and differences. Random errors in the estimates of blood pressure differences would be especially small, since every comparison is based on data from several thousand individuals per treatment group. Systematic errors were minimised by prespecifying the criteria for inclusion of trials, the comparisons to be studied, and the outcomes to be reported., ${ }^{4,11}$ Analyses were based on relative risk estimates rather than hazard ratios, but this does not seem to have substantially affected the findings: sensitivity analyses based on hazard ratios, previously published by contributing studies, identified no estimate of the pooled 
risk reduction that differed by more than one percent. The analyses were not adjusted for differences in achieved blood pressure or patients' characteristics, such as disease history or baseline blood pressure. Although a few outcome data were missing from some studies, subsidiary analyses suggest that this is unlikely to have had an important effect on the findings.

The analyses of heart failure reported here with data from 22 of the 29 studies, did not differ substantially from the findings of supplementary analyses including three additional trials that provided heart failure data based on a less restrictive definition than ours. Other supplementary analyses showed that these missing data were unlikely to have greatly affected estimates of treatment effects and treatment differences for total major cardiovascular events. Incomplete adherence to randomised treatments will, however, have resulted in substantial and systematic underestimation of both the effects of active treatments compared with control, and the differences between regimens based on different drug classes. ${ }^{4}$ In general, about two-thirds of participants remained on randomised treatment at the end of follow-up. Assuming uniform loss of compliance over time, the treatment effect that would have been seen with complete adherence would be about $20 \%$ greater than that noted.

Evidence of heterogeneity of outcome in contributing trials was seen for two comparisons, those of ACEinhibitor-based regimens versus calcium-antagonist-based regimens for coronary heart disease $(p=0.01)$ and, of regimens targeting different blood pressure goals for total mortality $(p=0.09)$. Heterogeneity in the comparison of ACE-inhibitor-based regimens versus calcium-antagonistbased regimens was mainly due to one trial, ${ }^{30}$ and neither exclusion of that trial from the fixed-effect model nor the use of a random-effect model in either comparison altered the conclusions. Stratification of the analyses by method of treatment allocation (double-blind or open) identified one comparison for which there was some evidence of heterogeneity $(p=0 \cdot 04)$; there was a trend towards a lower risk of coronary heart disease with diuretic or $\beta$-blockerbased regimens than with calcium-antagonist-based regimens in open trials ${ }^{35,39}$ but not in double-blind studies $^{18,25,29,31,36-38}$ However, exclusion of the open studies from the analysis did not alter the conclusions.

Stratification of drug regimens into major subgroups identified no significant heterogeneity. In the comparisons of calcium-antagonist-based regimens, there was no significant evidence of heterogeneity for any outcome between trials that used dihydropyridine agents and those that used non-dihydropyridine agents. In the comparisons of diuretic or $\beta$-blocker-based regimens with other regimens, there was no evidence of heterogeneity for any outcome between trials that used diuretic alone, those that used $\beta$ blockers alone, or those that allowed the use of either as initial treatment. However, investigators in one large trial ${ }^{32}$ reported reduced risks of cardiovascular events with a regimen based on the diuretic chlorthalidone, compared with that based on an ACE inhibitor.

Our results provide answers to questions about the effects of several commonly-used blood-pressure-lowering regimens for patients with high blood pressure or those otherwise at risk of major cardiovascular events. The findings are consistent with results of most of the large contributing trials, with small differences that might indicate the increased precision of our analyses, slight differences in treatment regimens, or differing ethnic backgrounds of participants. With respect to ethnic background, results in one large trial ${ }^{32}$ showed a smaller reduction of total major cardiovascular events with ACE- inhibitors than with diuretic; this seems to be due, at least in part, to the large proportion of African-American patients in the study and the much smaller reduction in blood pressure achieved with ACE inhibitor in that subgroup. However, although several questions are resolved by these analyses, others remain unanswered. For example, the findings of several individual studies ${ }^{13,17,29}$ indicate that some regimens might be of greater or lesser benefit for patients with established diseases, such as diabetes or nephropathy, or in outcomes that were not included in the current overviews, such as new-onset diabetes or renal failure.

We have sufficient data to do further subsidiary analyses ${ }^{11}$ that will address these and related questions. Planned analyses of the associations between blood pressure differences and risk reductions in trials of every drug class should also make clear the contribution of blood pressure lowering versus other drug-specific mechanisms to treatment outcomes. Until such findings are known, our results, assessed in the context of other relevant data ${ }^{49,50}$ and treatment guidelines, ${ }^{51,52}$ should enhance the ability of clinicians and health policy makers to make evidencebased decisions about the use of the different bloodpressure-lowering regimens currently available.

Blood Pressure Lowering Treatment Trialists' Collaboration Writing committee-F Turnbull, B Neal, C Algert, J Chalmers, M Woodward, S MacMahon.

Executive committee-S MacMahon, C Baigent, J Cutler, R Fagard, B Neal, P Whelton, S Yusuf.

Coordinating centre-C Algert, J Chalmers, N Chapman, S MacMahon, B Neal, F Turnbull, $M$ Woodward.

Other members-L Agodoa, C Baigent, H Black, J-P Boissel, B Brenner, M Brown, C Bulpitt, R Byington, J Chalmers, R Collins, J Cutler, B Dahlof, B Davis, J Dens, R Estacio, R Fagard, K Fox, L Hansson (deceased), R Holman, L Hunsicker, J Kostis, K Kuramoto, E Lewis, L Lindholm, J Lubsen, S MacMahon, E Malacco, G Mancia, B Neal, C Pepine, M Pfeffer, B Pitt, P Poole-Wilson, G Remuzzi, A Rodgers, P Ruggenenti, R Schrier, P Sever, P Sleight, J Staessen, K Teo, $\mathrm{R}$ Turner (deceased), P Whelton, L Wing, Y Yui, S Yusuf, A Zanchetti.

Conflict of interest statement

Of the writing committee members, JC, SM, BN, and MW have received travel expenses, payment for speaking at meetings, or research funding from one or more agencies that sponsored trials included in this collaboration.

\section{Acknowledgments}

We thank C Anderson, D Catellier, W Elliott, A Ferraro, L Elvelin, G Grandits, E Handberg, L Hemphill, J Lanke, H Merianos, B Mullane, C Palmer, J Pogue, S Pressel, D Ramjit, C Reid, I Stratton, L Thijs, $\mathrm{J}$ Wang, I Warnold, and K Willson for their comments and contributions to this report. The analyses in this report have been supported by grants and awards provided by the National Health and Medical Research Council of Australia. B Neal is the recipient of a fellowship from the National Heart Foundation of Australia. Meetings of collaborators for this cycle of analyses were sponsored by Bayer AG, Boehringer-Ingelheim, Merck, Pfizer, Servier, and Solvay.

\section{References}

1 Murray C, Lopez A. The global burden of disease. Cambridge: Harvard University Press, 1996.

2 WHO. World health report 2002; reducing risk, promoting healthy life. Geneva: World Health Organization, 2002

3 Gueffiyer F, Boutitie F, Boissel J-P, et al. Effect of antihypertensive drug treatment on cardiovascular outcomes in women and men: a meta-analysis of individual patient data from randomised controlled trials. Ann Intern Med 1997; 126: 761-67.

4 Blood Pressure Lowering Treatment Trialists' Collaboration. Effects of ACE inhibitors, calcium-antagonists and other blood pressure lowering drugs: results of prospectively designed overviews of randomised trials. Lancet 2000; 355: 1955-64.

5 Psaty B, Smith N, Siscovick D, et al. Health outcomes associated with antihypertensive therapies used as first-line agents: a systematic review and meta-analysis. $7 A M A$ 1997; 277: 739-45.

6 Yusuf S, Peto R, Lewis J, Collins R, Sleight P. Beta-blockade during and after myocardial infarction: an overview of the randomized trials. Prog Cardiovasc Dis 1985; 27: 335-71.

7 Garg R, Yusuf S. Overview of randomized trials of angiotensin- 
converting enzyme inhibitors on mortality and morbidity in patients with heart failure. FAMA 1995; 273: 1450-56.

8 Flather M, Yusuf S, Kober L, et al. Long-term ACE inhibitor therapy in patients with heart failure or left-ventricular dysfunction: a systematic overview of data from individual patients. Lancet 2000; 355: 1575-81.

9 Pahor M, Psaty B, Alderman M, et al. Health outcomes associated with calcium-antagonists compared with other first-line antihypertensive therapies: a meta-analysis of randomised controlled trials. Lancet 2000; 356: $1949-54$.

10 Psaty B, Lumley T, Furberg C, et al. Health outcomes associated with various antihypertensive therapies used as first-line agents. $f A M A$ 2003; 289: 2534-44

11 World Health Organization-International Society of Hypertension Blood Pressure Lowering Treatment Trialists' Collaboration. Protocol for prospective collaborative overviews of major randomized trials of blood-pressure lowering treatments. F Hypertens 1998; 16: 127-37.

12 Lithell H, Hansson L, Skogg I, et al. The Study on Cognition and Prognosis in the Elderly (SCOPE): principal results of a randomised double-blind intervention trial. F Hypertens 2003; 21: 875-86.

13 Lewis E, Hunsicker L, Clarke W, et al. Renoprotective effect of the angiotensin-receptor antagonist irbesartan in patients with nephropathy due to type 2 diabetes. $N$ Engl f Med 2001; 345: 851-60.

14 Brenner B, Cooper M, De Zeeuw D, et al. Effects of losartan on renal and cardiovascular outcomes in patients with type 2 diabetes and nephropathy. N Engl f Med 2001; 345: 861-69.

15 Dahlöf B, Devereux R, Kjeldsen S, et al. Cardiovascular morbidity and mortality in the Losartan Intervention For Endpoint reduction in hypertension study (LIFE): a randomised trial against atenolol. Lancet 2002; 359: 995-1003.

16 ALLHAT Officers and Coordinators for the ALLHAT Collaborative Research Group. Major cardiovascular events in hypertensive patients randomized to doxazosin vs chlorthalidone: the Antihypertensive and Lipid-Lowering Treatment to Prevent Heart Attack Trial (ALLHAT). fAMA 2000; 283: 1967-75.

17 Hansson L, Zanchetti A, Carruthers S, Dahlof B, Elmfeldt D, Julius S. Effects of intensive blood-pressure lowering and low-dose aspirin in patients with hypertension: principal results of the Hypertension Optimal Treatment (HOT) randomised trial. Lancet 1998; 351: 1755-62.

18 National Intervention Cooperative Study in Elderly Hypertensives Study Group. Randomized double-blind comparison of a calciumantagonist and a diuretic in elderly hypertensives. Hypertension 1999; 34: 1129-33.

19 MacMahon S, Sharpe N, Gamble G, et al. Randomised, placebocontrolled trial of the angiotensin converting enzyme inhibitor, ramipril, in patients with coronary or other occlusive vascular disease. f Am Coll Cardiol 2000; 36: 438-43.

20 Pitt B, Byington R, Furberg C, et al. Effect of amlodipine on the progression of atherosclerosis and the occurrence of clinical events. Circulation 2000; 102: 1503-10.

21 PROGRESS Collaborative Group. Randomised trial of a perindoprilbased blood pressure lowering regimen among 6105 individuals with previous stroke or transient ischaemic attack. Lancet 2001; 358: 1033-41.

22 Teo K, Burton J, Buller C, et al. Long-term effects of cholesterol lowering and angiotensin-converting enzyme inhibition on coronary atherosclerosis: the Simvastatin/enalapril Coronary Atherosclerosis Trial (SCAT). Circulation 2000; 102: 1748-54.

23 Staessen J, Fagard R, Thijs L, et al. Randomised double-blind comparison of placebo and active treatment for older patients with isolated systolic hypertension in Europe. Lancet 1997; 350: 757-64.

24 UK Prospective Diabetes Study Group. Tight blood pressure contro and risk of macrovascular and microvascular complications in type 2 diabetes: UKPDS 38. BMF 1998; 317: 703-13.

25 Zanchetti A, Agabiti-Rosei E, Dal Palu C, Leonetti G, Magnani B, Pessina A. The Verapamil in Hypertension and Atherosclerosis Study (VHAS): results of long-term randomised treatment with either verapamil or chlorthalidone on intima-media thickness. F Hyperten 1998; 16: 1667-76.

26 HOPE (Heart Outcomes Prevention Evaluation) Study Investigators Effects of an angiotensin-converting-enzyme inhibitor, ramipril, on cardiovascular events in high-risk patients. N Engl f Med 2000; 342: 145-53.

27 Pitt B, O’Neill B, Feldman R, et al. The QUinapril Ischaemic Event Trial (QUIET): evaluation of chronic ACE inhibitor therapy in patients with ischaemic heart disease and preserved left ventricular function. Am 7 Cardiol 2001; 87: 1058-63.

28 Dens J, Desmet W, Coussement P, et al. Usefulness of nisoldipine for prevention of restenosis after percutaneous transluminal coronary angioplasty (results of the NICOLE study). Am f Cardiol 2001; 87: 28-33.

29 Wright J, Bakris G, Green T, et al. Effect of blood pressure lowering and antihypertensive drug class on progression of hypertensive kidney disease: results from the AASK Trial. $f A M A$ 2002; 288: 2421-31.

30 Estacio R, Jeffers B, Hiatt W, Biggerstaff S, Gifford N, Schrier R. The effect of nisoldipine as compared with enalapril on cardiovascular outcomes in patients with non-insulin dependent diabetes and hypertension. N Engl f Med 1998; 338: 645-52.

31 Schrier R, Estacio R, Esler A, Mehler P. Effects of aggressive blood pressure control in normotensive type 2 diabetic patients on albuminuria, retinopathy and strokes. Kidney Int 2002; 61: 1086-97.

32 ALLHAT Officers and Coordinators for the ALLHAT Collaborative Research Group. Major outcomes in high-risk hypertensive patients randomized to angiotensin-converting enzyme inhibitor or calcium channel blocker vs diuretic. $\mathcal{F A M A} 2002$; 288: 2981-97.

33 Wing L, Reid C, Ryan P, et al. A comparison of outcomes with angiotensin-converting-enzyme inhibitors and diuretics for hypertension in the elderly. N Engl F Med 2003; 348: 583-92.

34 Hansson L, Lindholm L, Niskanen L, et al. Effect of angiotensin converting enzyme inhibition compared with conventional therapy on cardiovascular morbidity and mortality in hypertension: the Captopril Prevention Project (CAPPP) randomised trial. Lancet 1999; 353: 611-16.

35 Hansson L, Lindholm L, Ekbom T, et al. Randomised trial of old and new antihypertensive drugs in elderly patients: cardiovascular mortality and morbidity the Swedish Trial in Old Patients with Hypertension-2 study. Lancet 1999; 354: 1751-56.

36 Black H, Elliot W, Grandits G, et al. Principal results of the Controlled Onset Verapamil Investigation of Cardiovascular Endpoints (CONVINCE) trial. $\mathcal{F} A M A$ 2003; 289: 2073-82.

37 Zanchetti A, Bond M, Hennig M, et al. Calcium-antagonist lacidipine slows down progression of asymptomatic carotid atherosclerosis. Circulation 2002; 106: 2422-27.

38 Brown M, Palmer C, Castaigne A, et al. Morbidity and mortality in patients randomised to double-blind treatment with a long-acting calcium-channel blocker or diuretic in the International Nifedipine GITS study: Intervention as a Goal in Hypertension Treatment (INSIGHT). Lancet 2000; 356: 366-72.

39 Hansson L, Hedner T, Lund-Johansen P, et al. Randomised trial of effects of calcium-antagonists compared with diuretics and $\beta$ blockers on cardiovascular morbidity and mortality in hypertension: the Nordic Diltiazem (NORDIL) study. Lancet 2000; 356: 359-65.

40 Malacco E, Marcia G, Rapelli A, Menotti A, Zuccaro M, Coppini A for the Shell Investigators. Treatment of isolated systolic hypertension: the SHELL study results. Blood Press 2003; 12: 160-67.

41 Yui Y, Sumiyoshi T, Kodama K. Long-term effects of nifedipine retard vs ACE inhibitors in hypertension with coronary heart disease: final report of JMIC-B. Circ f 2002; 66 (suppl): 357 .

42 Woodward M. Epidemiology, study design and data analysis. London: Chapman and Hall, 1999.

43 Whitehead A, Whitehead J. A general parametric approach to the metaanalysis of randomized clinical trials. Stat Med 1991; 10: 1665-1677.

44 UK Prospective Diabetes Study Group. Efficacy of atenolol and captopril in reducing risk of macrovascular and microvascular complications in type 2 diabetes: UKPDS 39. BMF 1998; 317: 713-20.

45 Cohn J, Ziesche S, Loss L, Anderson G, and the V-HeFT Study Group. Effect of felodipine on short-term exercise and neurohormone and long-term mortality in heart failure: results of V-Heft III. Circulation 1995; 92: 1-143.

46 Carson P, O'Connor C, Miller A, et al. Circadian rhythm and sudden death in heart failure: results from Prospective Randomized Amlodipine Survival Trial. f Am Coll Cardiol 2000; 36: 541-46.

47 Ad Hoc Subcommittee of the Liaison Committee of the World Health Organization and the International Society of Hypertension. Effects of calcium-antagonists on the risks of coronary heart disease, cancer and bleeding. F Hypertens 1997; 15: 105-15.

48 MacMahon S, Collins R, Chalmers J. Reliable and unbiased assessment of the effects of calcium-antagonists: importance of minimizing both systematic and random errors. F Hypertens 1997; 15: 1201-04.

49 Collins R, Peto R, MacMahon S, et al. Blood pressure, stroke, and coronary heart disease: part 2 , short-term reductions in blood pressure: overview of randomised drug trials in their epidemiological context. Lancet 1990; 335: 827-39.

50 Collins R, MacMahon S. Blood pressure, antihypertensive drug treatment and the risks of stroke and of coronary heart disease. Br Med Bull 1994; 50: 272-98.

51 Guidelines Committee. European Society of Hypertension-European Society of Cardiology Guidelines for the management of arterial hypertension. F Hypertens 2003; 21: 1011-53.

52 Chobanian A, Bakris G, Black H, et al. The seventh report of the Joint National Committee on prevention, detection, evaluation and treatment of high blood pressure: the JNC 7 report. $\mathcal{F A M A} 2003$; 289: 2560-72. 